\title{
edmetic
}

Revista de Educación Mediática y TIC

Estrategias para la indagación continuada de trabajos académicos utilizando herramientas de Google. El caso de una investigación sobre infografías en la educación

Strategies for the continued inquiry of academic papers using Google tools. The case of an investigation on infographics in education

Fecha de recepción: 30/05/2017

Fecha de revisión: 11/06/2017

Fecha de aceptación: 12/06/2017 


\title{
Estrategias para la indagación continuada de trabajos académicos
}

utilizando herramientas de Google. El caso de una investigación sobre

infografías en la educación

\author{
Strategies for the continued inquiry of academic papers using Google \\ tools. The case of an investigation on infographics in education
}

\section{Gonzalo Abio'}

\section{Resumen:}

El desarrollo de la literacidad académica requiere el dominio de competencias informacionales y digitales, de forma que se consigan realizar los objetivos pretendidos en una investigación y poder participar de forma eficaz en las diversas prácticas sociales relacionadas. En este trabajo describimos el proceso realizado en una búsqueda bibliográfica sobre infografías en la educación en el cual nos valemos de recursos como el Google académico y el sistema de alertas del propio Google, para elaborar una base de datos que está en continuo crecimiento. Con base en la información reunida sobre el tema en cuestión, encontramos que, en comparación con los trienios anteriores analizados, en el trienio de 2014-2016 hubo un incremento significativo del número de trabajos que relacionan, desde diversas perspectivas, las infografías con la educación. Estimamos que las estrategias de indagación académica continuada que han sido empleadas en esta investigación pueden ser también útiles en los diversos ámbitos (personal, escolar, profesional o académico) de la vida de los estudiantes. Al final del texto se presenta una lista con recomendaciones para orientar la realización de este tipo de búsqueda bibliográfica.

Palabras claves: competencia digital, competencia informacional, Google académico, educación científica.

\begin{abstract}
:
The development of academic literacy requires the mastery of informational and digital competences, in order to achieve the desired objectives and to be able to participate effectively in the related social practices. In this paper, we describe the process carried out in a bibliographic search about infographics in education in which we use resources such as the Google Scholar and the own Google's alerts system to create a database that is constantly growing. Based on the information collected on the subject of study, we found that, in comparison with the previous triennia analyzed, in the three-year period 20142016 there was a significant increase in the number of works linking, from different perspectives, infographics with education. We believe that the strategies of continuous academic inquiry that have been used in this research
\end{abstract}

1 Universidade Federal de Alagoas, Brasil; gonzalo@cedu.ufal.br 
can also be useful in the various domains (personal, professional or academic) of students' lives. At the end of the text a list is presented with useful recommendations to guide the implementation of this type of literature search.

Keywords: digital competence, information skills, Google scholar, scientific education. 


\section{Introducción}

Entre lo social, lo semiótico y lo pedagógico hay una estrecha interconexión (Bezemer y Kress, 2016, p.viii), de forma que la transformación social y cultural en el uso de múltiples lenguajes y medios trae innúmeros desafíos para la educación actual y futura. Esa alteración profunda de la infraestructura tecnológica por los medios y lenguajes que aparecen y se desarrollan en estos últimos tiempos ejerce una presión para un cambio de paradigma educacional como no se había visto hasta ahora (Pérez Tornero, 2015).

Con las posibilidades tecnológicas actuales no hay límites para la educación, pues los muros de la escuela se derrumban y el aprendizaje es posible en todo momento y lugar, de forma que se codean el aprendizaje formal con otras formas de aprendizaje informales y no formales, abriéndose el camino para un proceso de aprendizaje más continuo y fluido (seamless learning) (Chan et al., 2006; Wong, Milrad y Specht, 2015), si es que así lo desea el usuario, guiado por su curiosidad o necesidad.

En lo que se refiere al trabajo específico con la información y el conocimiento, esas nuevas posibilidades tecnológicas impulsan a cambios paulatinos, pero inexorables, en los hábitos de lectura y de estudio, pues ya no se considera imprescindible memorizar datos y hechos, cuando es más fácil y rápido hacer uso de las prótesis digitales de memoria y de información que tenemos siempre a mano con los dispositivos digitales con los que podemos ser también potenciales produsuarios en la sociedad de la información (Sacristán, 2013). Por ejemplo, si en el año 2000 el innovador motor de búsqueda automática de Google era prácticamente desconocido, poco después, y muy rápidamente, se convirtió en una herramienta para búsqueda de información casi que imprescindible y naturalizada para muchos usuarios de Internet.

En realidad, es tal la importancia que tiene la lectura, la solución de problemas y el aprendizaje a partir de la información que existe en línea, que Kervin, Mantei y Leu (en prensa), recomiendan darle una centralidad mayor al trabajo con los textos en línea, aunque sin desdeñar los textos impresos.

De cualquier forma, pese a todos los avances y transformaciones, es bueno recordar que consumo de información no es lo mismo que generación de conocimientos, tal como nos lo señalan Coiro (2015) y muchos otros autores 
(ver Resende, 2013).

El acceso fácil y casi instantáneo a las ingentes cantidades de información que brinda la Internet actual, encierra, a su vez, algunas paradojas interesantes (Abio, 2013), entre ellas, la tendencia a caer en rutinas por la preferencia a ciertos temas y a las tareas que son más agradables o fáciles de hacer, además de una falta de concentración y mayor dispersión del sujeto por la realización de varias tareas al mismo tiempo, como también una mayor posibilidad de infoxicación por el desbordamiento cognitivo ante el potencial exceso de información. Además, la orfandad digital y la procrastinación son otras características que la vida digital moderna hace aparecer también (Millán, 2016), cuestiones estas, entre muchas otras, a las que los sistemas educativos deben dar atención para poder llegar a formar ciudadanos más capaces y preparados.

Según Cassany y Ayala (2008), la mayoría de los jóvenes están aprendiendo de manera informal un conjunto de habilidades y técnicas de manejo de información. Sin embargo, el riesgo de ese aprendizaje informal supone la falta de una guía u orientación sobre la riqueza pedagógica de los servicios de información y comunicación disponibles. Esto propicia que los jóvenes exhiban habilidades digitales incipientes o con una visión inadecuada del potencial pedagógico que realmente ofrecen esos servicios (Organista Sandoval et al., 2017).

En un estudio sobre el uso de los recursos lingüísticos en línea que los alumnos utilizan en sus prácticas cotidianas de lectura y escritura, Cassany (2016) identificó dos tipos de usuarios: un usuario básico, que emplea un recurso cualquiera de modo mecánico y acrítico, y otro, sofisticado y autónomo, que elige conscientemente el recurso según el contexto, muestra conocimientos y destrezas refinados y verifica los resultados obtenidos por medio de varias estrategias. El autor recomienda la conveniencia de identificar con más detalle esas estrategias, para lvego implementarlas en la instrucción formal.

En resumen, a pesar de las facilidades que existen para conocer y 
aprender más sobre cualquier cosa que deseemos a partir de la información disponible hoy en día en Internet, el profesor debe continuar siendo en su trabajo pedagógico el arquitecto del conocimiento y guía de sus alumnos, ahora, en estas nuevas condiciones y configuraciones donde las competencias, estrategias y habilidades para el trabajo con la información son tan importantes como los conocimientos que habitualmente se enseñan.

Para el desarrollo de la literacidad académica es necesario dominar las competencias informacionales y digitales necesarias, entre otras competencias, para conseguir realizar los objetivos pretendidos en una investigación y poder participar de forma eficaz en las diversas prácticas sociales relacionadas, de una manera activa, crítica, propositiva y ética.

Teniendo como base la realización de una búsqueda de la literatura existente sobre el uso de infografías en la educación, en este trabajo nos enfocaremos, especialmente, en la descripción de las acciones y procedimientos utilizados en esa búsqueda, en la cual nos valemos, entre otros recursos, de varias herramientas y posibilidades que ofrece Google, o sea, herramientas que entendemos que son o pueden ser de fácil acceso y utilización en todos los niveles educativos. También mostraremos las estrategias que pueden ser utilizadas para facilitar el desarrollo de una literacidad tecnológica, informacional y académica en los estudiantes como apoyo a las prácticas investigativas que se hagan necesarias.

\section{Revisión de la literatura}

\subsection{Competencias en la educación}

Existe una atención creciente a las competencias necesarias para el ciudadano del siglo XXI. De acuerdo con Escamilla (2011 apud García Llorente, 2015), en las escuelas se han de tener las competencias básicas como núcleo de referencia, para que puedan ser concretadas de manera curricular en los diferentes niveles. De hecho, las competencias ya forman parte del currículo en los sistemas educativos de diversos países (García Valcárcel Muñoz Repiso, 2016)

A diferencia de los contenidos específicos, las competencias clave o básicas son multifuncionales, pues permiten la realización y el desarrollo 
personal a lo largo de la vida, la inclusión y la participación como ciudadanos activos y el acceso a un puesto de trabajo en el mercado laboral (Domingo Farnós, 2017). Como continúa el mismo autor, esas competencias, a diferencia de los contenidos específicos, son trasferibles, pues se aplican en múltiples situaciones y contextos para conseguir distintos objetivos, resolver situaciones o problemas variados y realizar diferentes tipos de trabajos

Estas competencias son también transversales e interdisciplinares a las áreas y materias curriculares, porque su aprendizaje no es exclusivo de una de ellas. Además, son integradoras, porque combinan conocimientos ("saber"), destrezas ("hacer") y actitudes ("querer"); y son dinámicas, porque la competencia de las personas carece de límites en su crecimiento y se construye a lo largo de la vida (Domingo Farnós, 201 1, 2017).

Una definición de competencia es de naturaleza intrínsecamente compleja, pero de forma general, en el ámbito educativo, se puede entender como la capacidad para aplicar los conocimientos con eficiencia, eficacia y satisfacción sobre algún aspecto de la realidad personal, social, natural o simbólica (Hernández Suárez, Gamboa Suárez y Ayala García, 2014).

Como advierten Sierra y Arizmendiarrieta, Méndez Giménez y Mañana Rodríguez (2013), en sentido estricto no se enseñan o aprenden competencias, sino que se desarrollan capacidades que hacen, a quien realiza determinadas tareas, ser más competente. Lo que se aprende en realidad es el saber realizar diversas acciones que, integradas y puestas en práctica en diversos contextos, configuran una competencia.

Cuando pensamos en el trabajo con la gestión de la información y la construcción del conocimiento que el ciudadano del siglo XXI necesita, hay dos competencias que se evidencian como muy importantes. Ellas son la competencia digital y la informacional.

En el blog colombiano sobre competencias informáticas e informacionales organizado por Alejandro Uribe Tirado, aparece la siguiente definición:

Competencias informacionales: conocimientos, habilidades y actitudes que 
posibilitan a las personas o grupos a utilizar de manera eficiente y eficaz, crítica y ética, la información que facilitan las TIC y otras fuentes impresas, visuales, sonoras y demás (multimodal-multialfabetización), con el fin de generar conocimientos académicos, educativos, investigativos, laborales, profesionales, económicos, políticos, sociales, culturales, etc. Estas competencias interrelacionan los procesos de búsqueda, localización, selección, recuperación, organización, evaluación, producción y divulgación de información. La adquisición de estas competencias implica los procesos de enseñanza-aprendizaje que buscan facilitar lo que se ha denominado desarrollo de habilidades informativas, alfabetización en información o alfabetización informacional (Uribe Tirado y Castaño Muñoz, 2010)².

No son pocas las instituciones y sistemas educativos que han lanzado estándares con las competencias digitales e informacionales que los alumnos deben poseer al final de los ciclos escolares, así como sus docentes (INTEF, 2013, 2017; MINEDUC-Enlaces, 2013; Ministerio de Educación Nacional, 2013; UNESCO, 2008, 2011 ) entre otros, a los que podemos añadir los diversos trabajos realizados dentro del proyecto DigComp, como se puede ver en García Valcárcel Muñoz Repiso (2016).

También relacionado con lo anterior, y desde una perspectiva general, las prácticas investigativas son una de las prácticas sociales focalizadas que se proponen en la Base Nacional Comum Curricular que está en fase avanzada de elaboración por el Ministério da Educação de Brasil.

Las prácticas investigativas se refieren a la participación de los estudiantes en actividades relacionadas con la valoración, la construcción y la divulgación de saberes y conocimientos, priorizando géneros del discurso del ámbito de la divulgación científica. Las temáticas pueden ser de naturaleza interdisciplinaria, pero también, y especialmente, se propone que traten de las relaciones que los sujetos establecen con el uso y el aprendizaje de lenguas y con modos de usar el lenguaje para identificar, definir, comprender y resolver problemas en las diversas áreas del conocimiento y en diferentes culturas (Brasil, 2016, p.127).

Por su parte, desde la visión de la literacidad, Chávez González y Cantú Ortiz (2015) conciben la literacidad académica como las prácticas letradas, entendidas como prácticas socioculturales, que llevan a cabo los estudiantes

2 El destaque es del propio autor, para diferenciarlo de las TIC como medios para acceder a información, que formaría parte de las competencias informáticas, tal 
a partir de tareas o actividades contextualizadas en una institución académica (universidad o escuela), y con un propósito comunicativo específico, que evidencian el uso discursivo disciplinar que le es propio, de acuerdo a su formación académica. Dichas prácticas se expresan a través de géneros discursivos y tipos de textos académico-profesionales.

Según cada autor, existen posturas diferentes a respecto de la literacidad académica, pero es necesario señalar que "la literacidad no es una sola ni se aprende para siempre; es una serie de competencias que están en constante evolución y construcción, dependientes del contexto y de la situación" (Londoño Vásquez, 2015). Sin embargo, no podemos olvidar que cualquier práctica de lectura y escritura académica lleva emparejada la manipulación y uso efectivo de las referencias a los trabajos académicos utilizados, que es uno de los asuntos al que nos dedicaremos en este trabajo.

\subsection{Sobre infografías}

En el panorama de la comunicación y el aprendizaje que tiende a ser cada vez más multimodal, el uso de las infografías, junto con otras formas de visualización de datos e ideas, adquiere una importancia cada vez mayor, facilitado por su presencia común y creciente en las redes sociales y en los medios de comunicación impresos o digitales de amplia circulación (Confalone, 2012; Ranieri, 2008; Yekta, 2016).

De forma sencilla se puede decir que las infografías son representaciones diagramáticas con contenido informativo (Teixeira, 2014), que presentan generalmente en su estructura textos, símbolos, íconos, diagramas, dibujos, entre otros elementos, los cuales son harmónicamente jerarquizados e integrados para formar una pieza gráfica rica e instructiva desde el punto de vista de la comunicación (Menezes y Pereira, 2016).

Ranieri (2008) observa, con base en varios autores, que no existe un significado único, acabado o definido de lo que es una infografía, pero sí hay el consenso de que su objetivo debe ser el de facilitar la comprensión de

como aparece en el blog Ci2-Colombia (http://ci2-colombia.blogspot.com.br). 
hechos, procesos y datos.

El contenido más sintético mostrado en las infografías le da al lector/usuario el poder de visualizar y seleccionar la información de forma rápida, economizando tiempo y esfuerzo en la construcción de significado y de conocimiento. Ese reconocimiento más rápido de la información se debe al uso de elementos visuales y de diseño que, cuando son bien empleados, proporcionan una mejor legibilidad y reducción de la complejidad de la carga de información presentada. La visualización de datos e ideas por medio de infografías permite que la información compleja sea ofrecida para el lector/usuario de una forma que pueda ser más fácilmente comprendida y consumida (Smiciklas, 2012).

Por otro lado, no son pocos los autores que afirman que el trabajo con infografías aumenta el conocimiento de los lectores/usuarios: Valero Sancho (2009); Vanichvasin (2013); Lyra et al. (2016); Yildirim (2016); Çifçi (2016); Pisarenko y Bondarev (2016), Al-Mohammadi (2017), entre otros.

Bottentuit, Lisboa y Coutinho (2011), después de revisar la literatura específica, y sumando su experiencia propia en el trabajo con infografías, identificaron una serie de potencialidades para su uso en el contexto educativo, lo cual refuerza nuestro interés en ese género multimodal.

Con el objetivo de reunir informaciones sobre el tema de las infografías en la educación, realizamos una búsqueda bibliográfica con una serie de procedimientos que presentaremos y discutiremos a continuación.

\section{Descripción de la investigación realizada y resultados obtenidos}

Como base preliminar para una investigación de doctorado que está en ejecución en este momento, cuyo objetivo es la preparación y análisis de un curso sobre infografías destinado a los profesores brasileños de lenguas extranjeras, fue realizada una búsqueda de informaciones bibliográficas sobre infografías que tuvo varias fases.

La búsqueda inicial fue realizada en los primeros días de enero de 2014 en los currículos disponibles en el portal Lattes, ${ }^{3}$ que reúne informaciones

3 Disponible en: $\underline{\text { http://lattes.cnpq.br }}$ 
académicas de investigadores en Brasil, para lo cual fueron utilizados en el campo de búsqueda por asunto los términos "infográfico" e "infografia". Esta primera búsqueda proporcionó 83 y 141 currículos, respectivamente con algún tipo de mención a esos términos. Fue revisado cada uno de los currículos indicados y constatamos la escasez de publicaciones sobre el uso educacional de las infografías, prevaleciendo la mayor ocurrencia en el área de comunicación en el contexto nacional.

Vale señalar que dos nuevas búsquedas por asunto fueron implementadas en la misma plataforma Lattes. Una, en abril de 2016, mostró 333 y 384 ocurrencias para "infográfico" e "infografia", mientras que, en enero de 2017, fueron 388 y 509, respectivamente, números que son bien más altos que los encontrados en 2014 usando un procedimiento de búsqueda semejante.

Entre noviembre y diciembre de 2013 también fueron realizadas búsquedas sobre ese tema en la Biblioteca Digital de Teses e Dissertações (BDTD) ${ }^{4}$, así como de artículos académicos en la plataforma de Periódicos CAPES5, en los portales de los grupos editoriales Routledgé y SAGE7 y en la base de datos española DIALNET8, además de en el Google Académico (Google Scholar). En estas búsquedas también constatamos el poco número de trabajos específicos con infografías y visualización de datos realizados en el área de la educación. Esto no quiere decir que el tema no pudiera estar presente en otras producciones, principalmente del área de periodismo y comunicación, pero verificamos que existían en esos momentos pocas referencias sobre el uso educacional de infografías y visualización de datos.

En febrero de 2014 la lista, con 108 referencias seleccionadas y reunidas hasta ese momento sobre el tema en cuestión, fue colocada en un documento de Google drive abierto a las contribuciones y comentarios de los

\footnotetext{
${ }^{4}$ Disponible en: $\underline{\text { http://bdtd.ibict.br }}$

5 Disponible en: http://www.periodicos.capes.gov.br

6 Disponible en: https://www.routledge.com

7 Disponible en: https://us.sagepub.com

8 Disponible en: $\underline{\text { https://dialnet.unirioja.es }}$
} 
visitantes ${ }^{9}$ y también en un blog elaborado en Blogger. ${ }^{10}$ Esa lista fue divulgada para investigadores nacionales que se interesan por ese tema, de forma que, hasta ahora, el documento inicial ha aumentado con la colaboración de los especialistas y educadores, pero, principalmente, porque nuevos textos son incorporados en la medida en que son publicados, ya que el sistema de alertas del Google Académico fue configurado para enviar avisos automáticos cuando su motor de búsqueda encuentra nuevos textos publicados con las palabras clave de nuestro interés ("infographic", "infografía" e "infográfico")"1 .

Como resultado del trabajo continuado de localización, filtrado y divulgación de informaciones, en los primeros días de febrero de 2017 esa lista contenía 267 referencias generales sobre infografía y visualización de datos, de las cuales 139 trabajos (52\% del total reunido) guardan algún tipo de relación con la educación.

Cuando analizamos la distribución por año de ese segundo grupo de trabajos, podemos observar que en el último trienio (2014-2016) hubo un aumento significativo del número de trabajos académicos publicados en Brasil y en el mundo sobre el uso de infografías y visualización de datos que tienen interés para la educación (ver Tabla 1). También se observan concentrados en el último trienio, los pocos trabajos encontrados dedicados al uso de gráficos informativos en la enseñanza de lenguas extranjeras, que es nuestro objeto de estudio principal.

\footnotetext{
9 Disponible en:

https://docs.google.com/document/d/1Of8uA7R9UV FHIvll7puXxbrFyxja2ipuXrrmlkfKro/edit

10 Blog "Infográficos na Educação", disponible en: https://infograficosnaeducacao.blogspot.com

11 Gracias a los comentarios y sugerencias de los visitantes, fueron añadidas a la lista inicial 11 referencias adicionales. Ese número puede ser considerado bajo, si se compara al número de referencias que se obtuvo por medio de los avisos automáticos.
} 
Tabla 1- Evolución por trienio, en el intervalo entre 2005 y 2016, del número de trabajos publicados y encontrados sobre infografías y visualización de datos, que tienen relación con educación en general o con enseñanza de lenguas extranjeras. Fuente: elaboración propia.

\begin{tabular}{ccccc}
\hline & $2005-2007$ & $2008-2010$ & $2011-2013$ & $2014-2016$ \\
\hline $\begin{array}{c}\text { Trabajos que tienen } \\
\text { relación con }\end{array}$ & 3 & 20 & 33 & 67 \\
educación en general & 0 & 0 & 1 & 9 \\
\hline $\begin{array}{c}\text { Trabajos que tienen } \\
\text { relación con la } \\
\text { enseñanza de } \\
\text { Lenguas Extranjeras }\end{array}$ & 0 & & & \\
\hline
\end{tabular}

Es interesante señalar que, sin contar las referencias de trabajos publicados en 2017 que han comenzado a aparecer y que no fueron considerados en esta publicación, las acciones continuadas que se realizan en la gestión de la información sobre el tema en cuestión han permitido que, a la lista con 108 referencias, inicialmente publicada en febrero de 2014, se le haya podido añadir hasta febrero de 2017, 159 referencias adicionales; o sea, hubo un aumento de $148,1 \%$ sobre el número de trabajos encontrados en la búsqueda inicial.

\section{Discusión y conclusiones}

Es posible observar que existe una tendencia al aumento en los tres últimos años (2014-2016) del número de trabajos académicos que hacen referencia a infografías y visualización de datos y que tienen algún tipo de relación con el contexto educacional. Semejante tendencia se observa también, aunque en números absolutos menores, en los trabajos específicos encontrados que tienen relación con la enseñanza o aprendizaje de lenguas extranjeras con apoyo de las infografías.

El análisis cualitativo de esos resultados será motivo de otro trabajo, pero en esta oportunidad quisimos resaltar la importancia que tiene, a nuestro modo de ver, el uso de algunas estrategias de gestión de la información utilizadas en esta investigación, que en vez de ser transversal, se ha realizado de forma longitudinal y con la intención de tener un carácter de desarrollo permanente. 
En primer lugar, el uso del servicio de alertas del Google Académico con las palabras clave de nuestro interés se ha revelado de gran utilidad para mantener actualizada esa lista con las nuevas publicaciones que aparecen sobre el tema, pero esta no ha sido la única estrategia utilizada con esa finalidad. El hecho de participar en varias redes académicas (Research Gate ${ }^{12}$ y Academia.edu ${ }^{13}$ ) donde se facilita el contacto entre los investigadores, también ha sido muy útil para mantenerse al tanto de las nuevas publicaciones.

Aunque los alertas enviados, tanto por el Google Académico, como por las dos redes sociales académicas mencionadas, facilitan el conocimiento de nuevas publicaciones, el caudal de informaciones reunidas debe ser filtrado para poder seleccionar las referencias que son de nuestro interés. Las referencias seleccionadas son reunidas en la lista mencionada, que también tiene el propósito de que sea útil para otras personas interesadas en ese tema.

Otras estrategias también han sido útiles para encontrar nuevas referencias sobre el tema en estudio. Entre ellas, la atención que se le debe dar a las referencias importantes que aparezcan en los trabajos de interés, así como la atención y seguimiento a las citaciones de dichos trabajos por otros autores. El trabajo cruzado o complementado entre estas dos estrategias de búsqueda adicionales, que el propio Google Académico facilita al señalar los autores que citan el trabajo de interés, ha permitido añadir nuevas referencias útiles a las búsquedas de información realizadas anteriormente.

En la tabla 2 a continuación, hemos reunido algunas diferencias entre estas estrategias y las que se utilizan en una búsqueda bibliográfica común.

\footnotetext{
12 Disponible en: https://www.researchgate.net

13 Disponible en: https://www.academia.edu
} 
Tabla 2- Diferencias entre dos tipos de búsqueda de trabajos académicos: búsqueda común e indagación académica continuada. Fuente: elaboración propia.

\section{Búsqueda bibliográfica común \\ Indagación continuada de trabajos} académicos

- Predominantemente transversal (de corta duración).

- Uso de pocas fuentes de trabajos académicos.

- Poca atención a las novedades, con poco o ningún uso de sistemas de alertas o avisos sobre nuevas publicaciones de interés.

- Poca atención a las citaciones y trabajos relacionados.

- Actividad predominantemente individual, para consumo principalmente privado.
- Longitudinal (de larga duración o permanente) con filtrado constante de informaciones.

- Uso del mayor número posible de fuentes, incluyendo las redes sociales académicas.

- Atención a las novedades, con uso intenso de sistemas de alertas y avisos automáticos de nuevas publicaciones que han sido configurados con las palabras clave de interés.

- Atención a las citaciones (źquién cita a quién?) y a los trabajos relacionados.

- Actividad individual o preferentemente colaborativa, social, con resultados disponibles a todos los interesados.

Aunque la recopilación de bibliografía aquí descrita fue realizada a partir de una investigación de posgrado, estimamos que el conjunto de estrategias empleadas en la búsqueda, selección y divulgación continua de una base de datos, también puede ser útil en otros niveles educativos. Estas estrategias pueden ser aprovechadas como tema de estudio en la enseñanza de procesos y hábitos de indagación académica y divulgación de resultados para su uso en los diversos ámbitos (personal, escolar, profesional o académico) de la vida de los estudiantes.

Como observaron Martínez Ortega, Subías y Cassany en un reciente estudio (2016), para la formación digital escolar es necesario realizar dos tareas complejas: (1) la identificación de aquellas prácticas letradas digitales a las que se quiere incorporar a esos alumnos, y (2) la transformación de dichas prácticas letradas digitales en objetos de aprendizaje, donde los alumnos puedan experimentar las herramientas digitales sin disociarlas de las prácticas comunicativas que le dan sentido (Martínez Ortega, Subías y Cassany, 2016, pp. 209-210). 
Con esas tareas en mente y con base en la experiencia aquí descrita, para finalizar este trabajo hemos elaborado una lista con recomendaciones que pueden orientar y ayudar al desarrollo consciente de estrategias para aprovechar mejor la búsqueda y gestión de informaciones académicas durante la realización de las prácticas investigativas que pudieran ser propuestas a los estudiantes (ver Anexo 1).

Para estas recomendaciones hemos llevado en consideración algunos descriptores de las competencias referentes al área 1 (de información y alfabetización digital) y del área 2 (comunicación y colaboración) que han sido tomadas del marco general de competencia digital del INTEF (2017), así como otros descriptores específicos de la competencia investigativa que aparece en el documento del Ministerio de Educación Nacional colombiano (2013).

\section{Referencias bibliográficas}

ABIO, G. (2013). El papel del profesor de español en la era de las tecnologías y de la educación a distancia. Blog de Gonzalo Abio - E/LE. Recuperado de http://gonzaloabio-ele.blogspot.com.br/2013/04/el-papel-delprofesor-de-espanol-en-la.html

AL-MOHAMMADI, N. (2017). Effectiveness of Using Infographics as an Approach for Teaching Programming Fundamentals on Developing Analytical Thinking Skills for High School Students in the City of Makkah in Saudi Arabia. Global Journal of Educational Studies, 3(1), 22-42. Recuperado de http://www.macrothink.org/journal/index.php/gjes/article/view/10854

BEZEMER, J., y KRESS, G. (2016). Multimodality, learning and communication. A social semiotic frame. Oxon/New York: Routledge.

BOTTENTUIT Junior, J.B., LISBOA, E.S., y COUTINHO, C.P. (2011). O infográfico e as suas potencialidades educacionais. QUAESTIO. Revista de Estudos em Educação. 13(2), 163-183. Recuperado de http://periodicos.uniso.br/ojs/index.php?.journal=quaestio\&page=article\& op=view\&path\%5B\%5D=695

BRASIL (2016). Base Nacional Comum Curricular. 2a versão revista. Brasília: Ministério da Educação. Recuperado de 
http://www.consed.org.br/media/download/5733654a91c95.pdf

CASSANY, D. (2016). Recursos lingüísticos en línea: Contextos, prácticas y retos. Revista Signos. Estudios de Lingüística, 49 (supl. 1), 7-29. Recuperado de: http://www.scielo.cl/scielo.php?pid=S0718$09342016000400002 \&$ script $=$ sci abstract

CASSANY, D., y AYALA, G. (2008). Nativos e inmigrantes digitales en la escuela. Participación Educativa, 9, 53-71. Recuperado de https://dialnet.unirioja.es/servlet/articulo? codigo $=3105253$

CHAN, T.-W. et al. (2006). One-to-one technology-enhanced learning: An opportunity for global research collaboration. Research and Practice in Technology Enhanced Learning, 1(1), 3-29. Recuperado de https://www.sri.com/sites/default/files/publications/imports/G11 RPTEL.pdf CHÁVEZ GONZÁLEZ, G., y CANTÚ ORTIZ, L. (2015). La literacidad académica de los estudiantes universitarios. CIENCIA UANL. 18(71), 39-44. Recuperado de http://eprints.uanl.mx/3862/1/Ciencia\%20UANL\%2018\%2C71.pdf

COIRO, J. (2015). Purposeful, Critical, and Flexible: Vital Dimensions of Online Reading and Learning. En SPIRO, Rand J. et al., Reading at a Crossroads? Disjuntures and Continuities in Current Conception and Practices (pp.8698). New York/Oxon: Routledge. Recuperado de http://www.academia.edu/21601391/Coiro J.2015. Purposeful critical and flexible Key dimensions of online reading and learning. In Spiro et al Eds. Reading at a Crossroads Disjunctures and Continuities in Curr ent_Conceptions_and_Practices_pp._53-64_

CONFALONE, J. (2012). The rise of infographics. DMN. Recuperado de http://www.dmnews.com/dataanalytics/the-rise-ofinfographics/article/246247

ÇIFÇI, T. (2016). Effects of Infographics on Students Achievement and Attitude towards Geography Lessons. Journal of Education and Learning, 5(1), 154166. Recuperado de http://www.ccsenet.org/journal/index.php/jel/article/view/56252 DOMINGO FARNÓS, J. (2017). Las TIC modifican los itinerarios de aprendizaje. 
Blog Juandon. Innovación y conocimiento. Recuperado de https://juandomingofarnos.wordpress.com/2017/03/02/las-tic-modificanlos-itinerarios-de-aprendizaje/

DOMINGO FARNÓS, J. (2011). Todo sobre competencias básicas y las TIC. Blog Juandon. Innovación y conocimiento. Recuperado de https://juandomingofarnos.wordpress.com/2011/01/20/todo-sobrecompetencias-basicas-y-las-tic/

ESCAMILLA GONZÁLEZ, A. (2011). Las competencias en la programación de aula (v. II). Barcelona: Graó.

GARCÍA LLORENTE, H.J. (2015). Multialfabetización en la sociedad del conocimiento:competencias informacionales en el sistema educativo. Revista Lasallista de Investigación, 12(2), 225-241. Recuperado de: http://repository.lasallista.edu.co/dspace/bitstream/10567/1394/1/225241.pdf

GARCÍA-VALCÁRCEL MUÑOZ-REPISO, A. (2016). Las competencias digitales en el ámbito educativo. col. DDOMI. Monografías del Departamento de Didáctica, Organización y Métodos de Investigación. Universidad de Salamanca. Recuperado de https://gredos.usal.es/ispui/handle/10366/130340

HERNÁNDEZ SUÁREZ, C.A., GAMBOA SUÁREZ, A.A., y AYALA GARCÍA, E.T. (2014). Competencias TIC para los docentes de Educación Superior. En Congreso Iberoamericano de Ciencia, Tecnología, Innovación y Educación. Recuperado de http://www.oei.es/historico/congreso2014/memoriactei/837.pdf

INTEF (2017). Marco Común de Competencia Digital Docente. Instituto Nacional de Tecnología Educativas y de Formación del Profesorado. Ministerio de Educación, Cultura y Deporte. Gobierno de España. Recuperado de http://www.slideshare.net/educacionlab/marco-comnde-competencia-digital-docente-2017

INTEF (2013). Marco Común de Competencia Digital Docente. v 2.0. Instituto Nacional de Tecnologías Educativas y de Formación del Profesorado. Madrid: Ministerio de Educación, Cultura y Deporte. Gobierno de España. Recuperado de: 
http://educalab.es/documents/10180/12809/MarcoComunCompeDigiDo ceV2.pdf

KERVIN, L., MANTEI, J. y LEU, D. J. (en prensa). Repositioning Online Reading to a Central Location in the Language Arts. En D. Lapp, D. Fischer (Eds.) Handbook of Research on Teaching the English Language Arts, 4th ed. Recuperado de https://www.researchgate.net/profile/Don_Leu/project/RepositioningOnline-Reading-To-A-Central-Location-In-The-LanguageArts/attachment/57defe7108aed713c2d77263/AS:407772383465476@1474 231920909/download/Ch.14KervinetalRepositioningOnlineReading_Final.docx

LONDOÑO VÁSQUEZ, D.A. (2015). De la lectura y la escritura a la literacidad: Una revisión del estado del arte. Anagramas, 14 (26), 197-220. Recuperado de $\quad$ http://www.scielo.org.co/scielo.php? script=sci abstract\&pid=S1692$25222015000100011 \& \operatorname{lng}=e s \& n r m=i s o \&$ tlng=es

LYRA, K.T. et al. (2016). Infographics or Graphics+Text: Which Material is Best for Robust Learning? En Proceedings of the IEEE International Conference on Advanced Learning Technologies (ICALT). Recuperado de https://arxiv.org/ftp/arxiv/papers/1605/1605.09170.pdf

MARTÍNEZ ORTEGA, F.; SUBÍAS, J., y CASSANY, D. (2016). Acercamiento etnográfico a la alfabetización digital en un Instituto de Educación Secundaria Obligatoria en Barcelona. CPU-e. Revista de Investigación Educativa, 23, 190-215. Recuperado de http://revistas.uv.mx/index.php/cpue/article/view/2168

MENEZES, H.F., y PEREIRA, C.P. de A. (2016). O uso da cor como informação: um estudo de caso dos infográficos da revista Galileu. Blucher Design Proceedings, 2(9), 4686-4697. Recuperado de http://www.proceedings.blucher.com.br/article-details/o-uso-da-corcomo-informao-um-estudo-de-caso-dos-infogrficos-da-revista-galileu$\underline{24639}$

MILLÁN, J.J. (2016). Huérfanos digitales. Abordaje multifactorial. Blog de Juan 
José Millán. Recuperado de http://blog.juanjosemillan.es/huerfanosdigitales

MINEDUC- ENLACES (2013). Habilidades TIC para el aprendizaje. Ministerio de Educación. Gobierno de Chile/ Enlaces. Recuperado de: http://historico.enlaces.cl/tp enlaces/portales/tpe76eb4809f44/uploadlm g/File/PDF/formacion/Matriz\%20de\%20Habilidades\%20TIC\%20para\%20el\% 20Aprendizaje.pdf

MINISTERIO DE EDUCACIÓN NACIONAL (2013). Competencias TIC para el Desarrollo Profesional Docente. Bogotá, Colombia. Recuperado de: http://www.colombiaaprende.edu.co/html/micrositios/1752/articles318264 recurso tic.pdf

ORGANISTA SANDOVAL, J., LAVIGNE, G., SERRANO SANTOYO, A. y SANDOVAL SILVA, M.I (2017). Desarrollo de un cuestionario para estimar las habilidades digitales de estudiantes universitarios. Revista Complutense de Educación, 28(1), 325-343. Recuperado de https://revistas.ucm.es/index.php/RCED/article/view/49802

PÉREZ TORNERO, J.M. (2015). El nuevo paradigma de la comunicación en la educación (I). Recuperado de https://imtornero.files.wordpress.com/2015/06/1-el-nuevo-paradigma-dela-comunicacic3b3n-en-la-educacic3b3nc2a0.pdf

PISARENKO, V., y BONDAREV, M. (2016). Infographics Use in Teaching Foreign Languages for Specific Purposes. Recent Patents on Computer Science, 9(2), 124-132.

RANIERI, P.R. (2008). A infografia digital animada como recurso para transmissão da informação em sites de notícia. Prisma.com, 7, 260-274. Recuperado de http://revistas.ua.pt/index.php/prismacom/article/view/673/pdf

RESENDE, T. de F. (2013). "Aprender a conhecer" na sociedade da informação: o papel específico da escola e os seus desafios. Revista de la Asociación de Sociología de la Educación, 6(2), 289-301. Recuperado de https://ojs.uv.es/index.php/RASE/article/view/8359

SACRISTÁN, A. (2013). Sociedad del conocimiento. En A. Sacristán (Comp.), Sociedad del conocimiento, tecnología y educación (pp.19-72). Madrid: 
Ediciones Morata.

SIERRA Y ARIZMENDIARRIETA, B., MÉNDEZ-GIMÉNEZ, A., y MAÑANA-RODRÍGUEZ, J. (2013). La programación por competencias básicas: hacia un cambio metodológico interdisciplinar. Revista Complutense de Educación, 24(1), 165-184.

Recuperado

de

https://revistas.ucm.es/index.php/RCED/article/viewFile/41196/39397

SMICIKLAS, M. (2012). The Power of Infographics. Using pictures to communicate and connect with your audience. Indiana: Que.

TEIXEIRA, C.C. da C. (2014). Criatividade, Design Thinking e Visual Thinking e sua relação com o universo da infografia e da visualização de dados. Tesis (doctorado)-Pontifícia Universidade Católica do Rio de Janeiro, Departamento de Artes e Design. Recuperado de http://www.maxwell.vrac.pucrio.br/Busca etds.php?strSecao=resultado\&nrSeq=24565@1

UNESCO (2011). Alfabetización Mediática e Informacional, Curriculum para Profesores. Recuperado de

http://unesdoc.unesco.org/images/0021/002160/216099S.pdf

UNESCO (2008). Estándares de Competencias en TIC para Docentes. Recuperado de http://www.oei.es/tic/UNESCOEstandaresDocentes.pdf

URIBE TIRADO, A., Y CASTAÑO MUÑOZ, W. (2010). La formación en competencias informáticas e informacionales desde la Escuela Interamericana de Bibliotecología con el apoyo una plataforma de elearning. Experiencias y resultados. Hélice: Revista Venezolana de Ciencias de la Información, 2(2), 1-28. Recuperado de http://www.cidtec.luz.edu.ve/images/stories/helice/v2n2/mejores_practic as/uribe y castano $\mathrm{mp} v 2 \mathrm{n} 2 . \mathrm{pdf}$

VALERO SANCHO, J.L. (2009). La transmisión de conocimientos a través de la infografía digital. Ámbitos, 18, 51-63. Recuperado de http://www.redalyc.org/articulo.oa?id $=16812722004$

VANICHVASIN, P. (2013). Enhancing the Quality of Learning Through the Use of Infographics as Visual Communication Tool and Learning Tool. En 
Proceedings ICQA 2013. International Conference on QA Culture: Cooperation or Competition, (pp.135-142). Bangkok: The Office for National Education Standards and Quality Assessment. Recuperado de http://www.icaa2014.com/downloads/Proceeding_29.pdf\#page=135 WONG, L.H., MILRAD, M., y SPECHT, M. (Eds) (2015). Seamless Learning in the Age of Mobile Connectivity. London: Springer.

YEKTA, N.J. (2016). Online Infographics. IJBPAS, 5(7), 1698-1706. Recuperado de: http://ijbpas.com/pdf/2016/July/1467221352MS\%20IJBPAS\%202016\%20380 $\underline{1 . p d f}$

YILDIRIM, S. (2016). Infographics for Educational Purposes: Their Structure, Properties and Reader Approaches. TOJET, The Turkish Online Journal of Educational Technology, 15(3), 98-110. Recuperado de http://www.tojet.net/articles/v15i3/15311.pdf

\section{Cómo citar este artículo:}

Abio, Gonzalo (2017). Algunas estrategias para la indagación continuada de trabajos académicos utilizando herramientas de Google. El caso de una investigación sobre infografías en la educación. EDMETIC, Revista de Educación Mediática y TIC, 6(1), 209-231. doi: https://doi.org/10.21071/edmetic.v6i2.6934 
Anexo 1- Lista de recomendaciones para una búsqueda bibliográfica sobre un tema académico por parte de los estudiantes.

Estas recomendaciones te pueden servir en caso de que tengas que hacer una búsqueda bibliográfica de trabajos académicos sobre un tema determinado. Léelas y discútelas con tus compañeros de clase y con el profesor o profesora:

- En las búsquedas de trabajos académicos es mejor que utilices el Google Académico en lugar del buscador de Google común;

- Aprende la función de algunos operadores booleanos que pueden ayudarte a encontrar más fácil lo que buscas. ${ }^{14}$

- Observa que en los resultados brindados por el Google Académico aparecen también las citas, o sea, otros autores y trabajos que hacen referencia al artículo señalado. Puedes visitar también esos trabajos más recientes para saber si son de interés o no, según tus propósitos;

- Cuando uses el Google Académico, observa que debajo de cada referencia hay la posibilidad de ver también la forma correcta de citar ese trabajo, según varias normas de citación académica que existen;

- Si instalas en el navegador web el botón del Google Académico tendrás un acceso más rápido o directo a los artículos académicos siempre que lo necesites (funciona en los navegadores Firefox y Chrome).

- Para que te mantengas siempre actualizado sobre el tema que estás buscando, puedes crear un alerta en el Google con las palabras clave o tema o publicación de interés. Puedes cruzarlo también con autor, por ejemplo, tema + autor, nombre del libro + autor, etc. ${ }^{15}$

- Conoce algunos repositorios de trabajos académicos y sitios web donde puedes buscar también trabajos académicos (Dialnet, Redalyc, DOAJ, Scielo, Portal de Periódicos CAPES, TESEO, BDTD, Research Gate, Academia.edu, etc.). Observa si existen servicios de alertas en esos sistemas y úsalos a tu favor.

- Reúne en alguna lista o sistema de gestión de referencias bibliográficas las referencias que son útiles para tus propósitos. Esa lista de referencias puede ser divulgada en diversas plataformas virtuales (blog, wiki, sitio web, red social, etc.) y también puede ser dejada como un documento colaborativo.

- Intenta involucrar a otras personas en ese trabajo de búsqueda, filtrado y publicación de referencias. La lista de referencias sobre un tema determinado será mucho más útil si es construida con la participación o comentarios de otras personas que se interesan también por ese tema.

\footnotetext{
${ }_{14}$ Consulte operadores booleanos o cómo refinar las búsquedas en Google https://www.google.com/intl/es/scholar/refinesearch.html

${ }_{15}$ Consulte https://support.google.com/websearch/answer/4815696?hl=es
} 